\title{
Mapping a hospital using OpenStreetMap and Graphhopper: A navigation system
}

\author{
K. A. F. A. Samah, S. Ibrahim, N. Ghazali, M. Suffian, M. Mansor, W. A. Latif \\ Faculty of Computer and Mathematical Sciences, Universiti Teknologi MARA, Malay sia
}

\begin{tabular}{|c|c|}
\hline Article Info & ABSTRACT \\
\hline Article history: & \multirow{11}{*}{$\begin{array}{l}\text { We present a mobile-based navigation system with the emphasis } \\
\text { on the pedestrian navigation for Hospital Kuala Lumpur (henceforth, HKL) } \\
\text { named as HKLNS. This practical research aimed to provide support and help } \\
\text { for the people who come to the HKL. Despite having signages, layouts } \\
\text { and other types of guidance to help them navigate, they might not be } \\
\text { sure their current location and the destination's paths. By using } \\
\text { the OpenStreetMap (OSM) and Graphhopper API as a solution, it managed } \\
\text { to help people to navigate the surrounding of the HKL. The findings } \\
\text { showed that users were able to know their current location and the system } \\
\text { generated the shortest pedestrian paths to the desired location using both } \\
\text { mappings. The functionality testing and experimental results surrounding } \\
\text { HKL demonstrated the effectiveness of this approach. The HKLNS } \\
\text { was reliable based on the functionality and validation testing conducted } \\
\text { towards } 5 \text { respondents with } 5 \text { different case study, in which it manage } \\
\text { to reduce more than } 50 \% \text { time taken with the t-test result of } 0.00041 \\
\text { ( } p<0.05 \text { ), indicated that the system was accepted. }\end{array}$} \\
\hline Received Oct 31, 2019 & \\
\hline Dec 28, 2019 & \\
\hline Accepted Feb 11, 2020 & \\
\hline Keywords: & \\
\hline GPS & \\
\hline Graphhopper & \\
\hline Hospital Kuala Lumpur & \\
\hline Navigation & \\
\hline OpenStreetMap & \\
\hline Outdoor navigation & \\
\hline
\end{tabular}

This is an open access article under the CC BY-SA license.

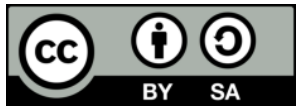

Corresponding Author:

K. A. F. A. Samah,

Faculty of Computer and Mathematical Sciences,

Universiti Teknologi MARA Cawangan Melaka Kampus Jasin,

77300 Merlimau, Melaka, Malaysia.

Email: khyrina783@uitm.edu.my

\section{INTRODUCTION}

Navigation is a science of coordinating a path by deciding its position, course, distance travelled and concerned in finding the right path to the desired location [1]. There are several types of navigation such as indoor navigation and outdoor navigation. As a general knowledge, outdoor navigation implements Global Positioning System (GPS) where it uses the satellite system to receive information about location and time. Whereas, indoor navigation uses Indoor GPS which benefits users as it has lower power consumption and smaller size requirements of wireless access devices, such as mobile phones and handheld computers. This study was focusing on the outdoor navigation.

Hospitals are usually huge, complex and changing spaces, which are regularly rearranged and extended as operational needs shift and change, often resulting in a confusing, non-well-thought-out layout and also often located within dense built-up areas, making the trip through and to the hospital a navigational challenge [2]. Hospital Kuala Lumpur (HKL) was chosen as the scope of study as it has a large area and many buildings. HKL is found on 150 sections on mainland and has 54 diverse divisions and units. These incorporate 29 clinical divisions and 15 clinical support administrations. The departments are located at different buildings and far from one another. HKL is always filled with lots of patients, staffs and visitors. The target of this research was mainly the visitors who went to HKL for an appointment for treatment 
and visiting patients. Established hospitals like HKL apparently are still under development bringing changes to the layout, location, and the name of the buildings, thus navigating these spaces can be a major test [3].

Recently, due to HKL's large area and extensive renovation programme, visitors faced problems to navigate themselves to the desired departments surrounding in HKL [4]. Firstly, despite being at the hospital frequently or occasionally, yet visitors were still unaware of the surrounding departments at HKL. Furthermore, although getting familiar with certain environments, the navigational trouble of a person's first encounter could last for a longer time for HKL visitors [5]. In addition, visitors were confused on which route to take to their desired departments from their current location [6]. It was also asserted that visitors searching for a shorter path became confused when the paths were too complex with interconnections. Consequently, as claimed by [7], with the lack of information provided, it can cause the visitors to arrive late for the appointments [8] and thus shortens the visiting hours [9]. Therefore, this HKL navigation system (HKLNS) is developed to navigate visitors of HKL to the desired departments and reduce the time taken to arrive there.

The difference between this system and other existing applications such as Waze and Google Maps is that this system focuses on the pedestrian route. Waze is all about contributing benefits to drivers out there on the road. Waze helps connecting drivers to one another that work together to improve the quality of everyone's daily routines. Drivers type their destination's address and drive with the app open while they passively contribute traffic and other road data [10]. Google Maps helps users to navigate the road accurately as it is based on real-time traffic information and even suggests which lane to be in [11].

Therefore, the main objective of this paper was to develop a mobile-based department's navigation system for HKL, to test the functionality and validation of the system effectiveness. OpenStreetMap (OSM) and Graphhopper API is applied in the system by mapping HKL as to determine the shortest and accurate route starting from user's current location to their desired departments. Besides the navigation as the outcome, visitors were able to know their current location, the location of the desired departments and navigate them towards the accurate route [12] in shorter time duration. Furthermore, this system has some extra features that users can find it very useful. Users can check any current changes on departments and routes. In addition, users can know such information from a list provided from the system. For users to receive such information, the admins will have to update the changes and they can do it from the system itself. The admins can also send notifications to the users to alert them for any changes.

This paper is organized as follows. In section 1, the researcher reviewed some background and literature of the developed systems. The related work is explained in section 2 followed by methodology in section 3. The results and discussion are presented in section 4 and finally section 5 is for the conclusion of the paper.

\section{RELATED WORK}

In this section, we describe the outdoor navigation, Global Positioning System (GPS) and the applications involved which are OpenStreetMap (OSM) and Graphhopper API.

\subsection{Outdoor navigation}

Outdoor navigation can be easily defined as navigating in an outdoor environment. These days, there are lots of people who use outdoor navigation systems when they want to reach the desired locations. For instance, people use Waze-GPS, Maps, Traffic Alerts \& Live Navigation (Waze) and Maps-Navigation $\&$ Transit (Maps) to navigate themselves to their desired locations. GPS has been the most important navigation system used for navigating in outdoor environment and has been widely implemented in mobile navigation applications [13].

However, it has been a problem for outdoor navigation systems to be mature as the outdoor environments are becoming more complex and unpredictable in terms of the nature of the route [14]. Thus, this study focused on the outdoor navigations since the study aimed to navigate users to their desired departments that were located at different buildings and were far apart at the HKL. Next section will cover on GPS, a technique to detect current location.

\subsection{Global positioning system}

The Global Positioning System or GPS was used to detect the user's current location. GPS can be used everywhere es pecially on the mobile phone, the smart grid and satellite orbit determination [15]. GPS is a navigation network that uses satellite system to get the position of an individual and the individuals can use GPS receivers to locate their current position and pinpoint their desired location [16, 17]. GPS searches and calculates the location and position of a person at a certain area in terms of longitude and latitude including the altitude [18]. 
There are some reasons as to why GPS is used widely in most devices in this world today. Firstly, the use of GPS can help in reducing cost as it is affordable and easy to install [15]. Secondly, GPS helps to provide exact, real-time, three-dimensional position and speed information coupled with accurate timing data [19]. As [20] claimed the same thing where GPS provides wide-world coverage and better accuracy compared to other applications such as Global Navigation Satellite Systems. In addition, GPS gives a real time data of the user's current position no matter where they are as long as their device has the GPS receiver.

Despites the GPS application on the smart phones shows the navigation maps do require the internet connection to perform well [20], yet this research focused on outdoor environment at HKL where it is located in the city, the internet availability was everywhere and could be easily accessed. This could definitely confirm that users could locate their current location perfectly. The next section discusses the meaning of API since OSM API and GraphHopper API were implemented in this study.

\subsection{Application programming interface}

The application programming interface, also known as API is used to implement the map and the navigation route in the application. API provides interface to allow application to access the service from the cloud service providers since the software they created did not meet the requirement for some of those who are in need of the software [21]. API is also a stable and scalable programmable data delivery mechanism [22]. API is vital in giving sorted out techniques to permit diverse registering preparing steps and tasks to convey in this way, a well-structured APIs are along these lines important to utilize the overall varieties of processing gadgets to isolate the capacities engaged with performing calculations, putting away and moving information between areas, managing and arranging its utilization, and constraining and securing access [23]. A map fetched from OpenStreetMap (OSM) API and for routing, GraphHopper API was used and will be explained further.

\subsubsection{OpenStreetMap}

OSM is referred as the Wikipedia map of the world; it is a volunteer-driven, internationally distributed association whose individuals work to make a common digital map of the world [24]. It aims to build and maintain a free editable map database of the world so that users do not need to pay for geodata and be subjected to restrict copyright and commitment authorization [25]. OSM has been one of the most successful Volunteered Graphical Information (VGI) projects in recent years, which has also been frequently cited in the Geographic Information System (GIS) community and have actually coordinated the creation, production and distribution of free mapping resources to support humanitarian relief efforts in many places around the world since 2009 [26].

In addition, OSM has been shown to have more complete geodata in a certain part of area and more locally and semantically accurate than other datasets [25]. Thus, this shows how usage of OSM API is crucial for this research to achieve its objectives. The mapping process OSM is structured in a manner as claimed by [26] where users can sign up and login, create or edit or delete the map, access the entire dataset, and retrieve the entire dataset history free-of-charge so that users can retrace back any action done.

\subsubsection{GraphHopper}

GraphHopper is a routing engine for routing planning. GraphHopper acts as an open source libraries for road network travel time estimation and solving static vehicle routing problems [27]. GraphHopper is a fast, efficient routing library that implements multiple routing algorithms, that has from two up to five GPS locations, can calculate the fastest or shortest route between those points and the fastest or shortest route is calculated based on speed limits or distance tags associated with OSM routes such as streets, roads, and pedestrian routes [28].

GraphHopper is chosen as it is expandable, tested well, and handles altitude profile easily without modifications and Graphhopper also supports unidirectional Dijkstra, one-to-many Dijkstra and uni-and bidirectional $\mathrm{A}^{*}$ where the algorithms calculate a weighting value for each route and user will receive the route with lowest weighting value [28-30]. Graphhopper also uses the Contraction Hierarchies (CH) algorithm to expedite common Dijkstra algorithm calculations, however, $\mathrm{CH}$ within Graphhopper are adjusted to find single A to B route pairs, not filling a matrix of travel times to and from man y locations [27]. Regardless of that, in terms of performance, GraphHopper works well because of speed due to its catching strategy where it is able provide routes over long distance which is over one hundred km very fast [28].

\section{RESEARCH METHOD}

In this study, the research method is divided into two phases; gathering the information and designing and developments. 


\subsection{Gathering information}

A survey was conducted to the HKL visitors and the researchers managed to get 76 respondents. All the respondents came from different backgrounds and different reasons for going to HKL. In general, $89.5 \%$ from the respondents agreed they did not know the specific department's surroundings in HKL, despite 16 of respondents had visited HKL for more than 2 times. At the same time, $82.9 \%$ of them agreed that they had experienced confusion on which route that they should take to reach the desired department. Figure 1 shows the distribution of methods used to search for certain buildings in HKL.

Longer time taken is reflected by lack of information due to the prediction path not being provided. This was proven, even though the method was available, $86.8 \%$ respondents claimed that they took longer time to go to reach the desired department, $82.9 \%$ of them agreed that they were late for the appointment with the doctor and $85.5 \%$ voted that consequences of it, the visiting hours were limited. In addition, due to lack of accurate location when moving independently, it caused them to be unaware of their current location [29]. As a response, people in the big building might get lost if there was no proper way to navigate them to their destination. This could cause them to be panic when they could not find the department and worried of getting late to for the appointment. Thus, this research was proposed in order to help the public arrive in the shortest possible time to the desired places.

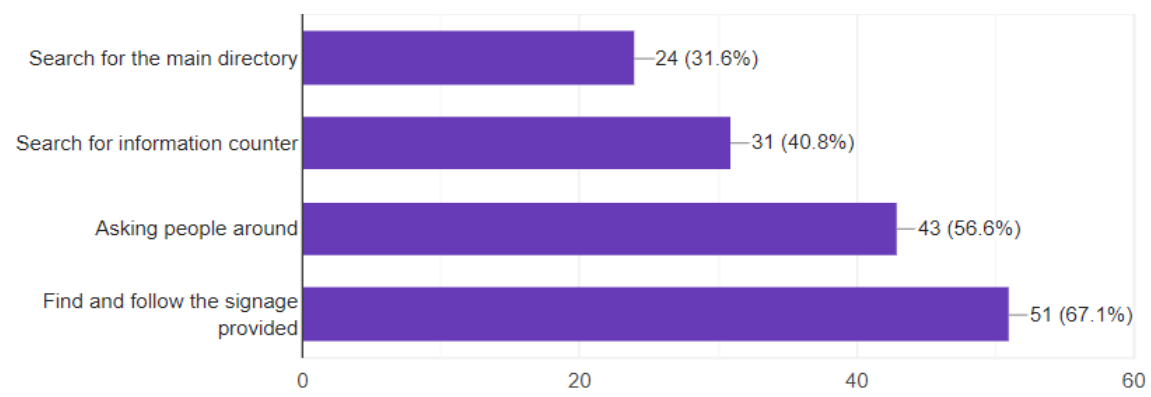

Figure 1. Methods used to search desired departments in HKL

\subsection{Design and development}

In this phase, four activities were involved to cater for the objective on mapping the HKL using OSM and Graphhopper API.

\subsubsection{Map editing of HKL}

This research only focused on the outdoor environments of HKL, specifically, on the pedestrian walk on the outdoor environment that would help the users on navigating from their current locations to the desired departments. Website OSM acts as a tool and it has an Editing API where it can fetch and save raw geodata from and to the OSM database. Figure 2 shows the webpage that was edited showing the existing map of HKL. Several steps were done when editing the map such as the lines from one point to another, set the "Foot Path" to separate the road type from the others, set the "Point" button to put marker on the building from the map and set it as "Hospital Ground" and named it according to the name of the building.

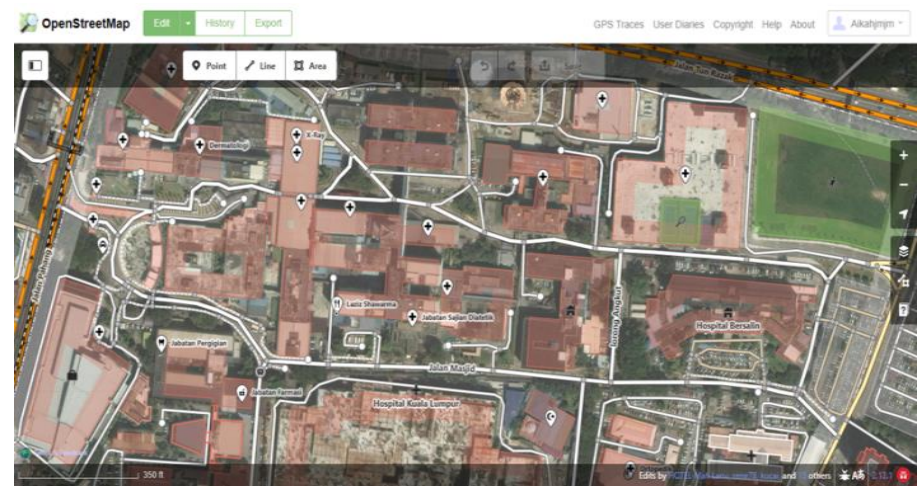

Figure 2. Webpage to edit map of HKL 


\subsubsection{Graphhopper API}

Once the editing was done, the save button was clicked and the map was updated. The changes however needed a period of time to see the result of the edited changes. Since OSM API is used in the system, the right code must be implemented so that the users were able to navigate to the desired departments based on the results generated from the application. To generate the polyline that would overlay on the map, GraphHopper's API was used as it is able to calculate and determine which route is suitable for the user to use. Figure 3 shows the flowchart of how GraphHopper's algorithm works. The function is to preprocess the graph such that subsequent shortest path queries can be processed quickly yet still provably correct. This was done with arranging the nodes by importance value and then replacing shortest paths through nodes with new shortcut edges. A faster shortest path search can be obtained by running a bidirectional shortest-path search making sure that the forward direction only traverses edges going to more important nodes and the backward direction only traverse edges coming from more important nodes.

\subsubsection{Flowchart diagram}

Figure 4 shows the flowchart diagram for users. Users did not need to login, all they had to do was to choose their desired destination. The system would generate a polyline along the chosen route thus, would help the users to navigate to their desired department.

\subsubsection{Functionality and validation of system}

For the functionality of the proposed system, each of the use case was tested in order to check for any any errors, bugs, or glitches that could be found in the system once it was conducted. Once all the problems were found, any problems found in the system were fixed and then tested again. The validation testing (VT) of the system were tested to compare the time taken to reach the desired department before and after using the proposed system. There had been 5 respondents with 5 different case study chosen to validate the systemeffectiveness and few steps were involved based on Table 1.

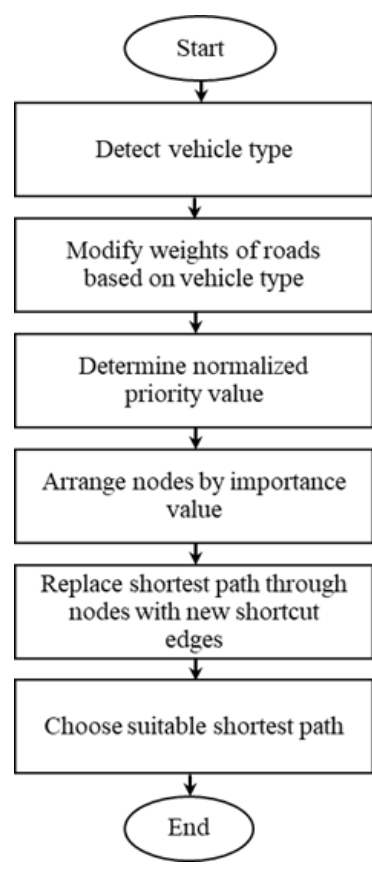

Figure 3. GraphHopper's algorithm flowchart

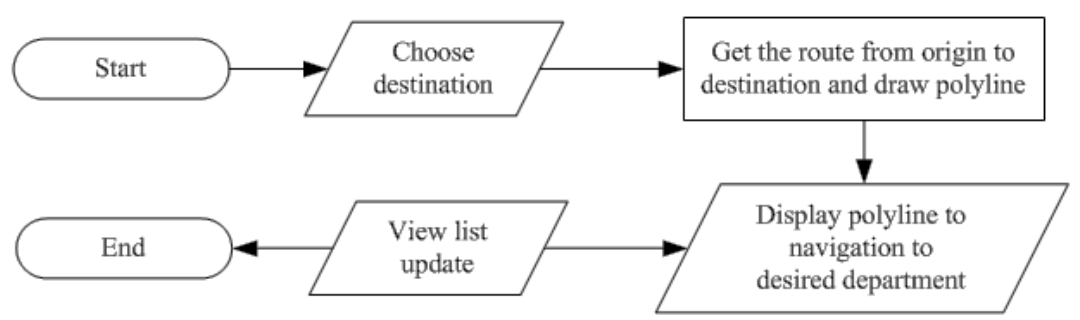

Figure 4. Flowchart diagram for user

Table 1. Activities for system validation

\begin{tabular}{cll}
\hline Method & & \multicolumn{1}{c}{ Steps of Activities } \\
\hline & 1) & Locate respondent's current location and set the desired location as \\
Before & in Table 3. \\
(without & 2) & Set the desired department \\
HKLNS) & 3) Start the stopwatch and respondent has to find own navigation \\
& 4) Once arrived, stop the stopwatch and record the time taken \\
& 1) Locate respondent's current location and set the desired location as \\
After & 2) User open HKLNS and set the desired department \\
(with HKLNS) & 3) Start the stopwatch and respondent follow the navigation \\
& 4) Once arrived, stop the stopwatch and record the time taken \\
\hline
\end{tabular}

\section{RESULTS AND ANALYSIS}

This section discusses the results and findings gained from the research for outdoor navigation by mapping HKL using OSM and Graphhopper.

\subsection{Functionality testing}

The functionality of the system was tested five times (Test 1 until Test 5) based on the menu provided in the prototype to make sure the function meets the requirement and works correctly for the user, 
and the result is depicted in Table 2. Figure 5 shows the snapshot of the HKLNS main menu which start with system detecting user's current location as in Figure 6 and user can select the desired location as in Figure 7. Figure 8 shows the selection menu followed by Figure 9 displays option for the admin in charge to update any information and finally Figure 10 shows the example of any latest news updated that was prompted in HKLNS.

Table 2. Functionality test result

\begin{tabular}{lccccc}
\hline \multicolumn{1}{c}{ Component } & Test 1 & Test 2 & Test 3 & Test 4 & Test 5 \\
\hline Select “User" button and appear current location & OK & OK & OK & OK & OK \\
Select "Choose Destination" & OK & OK & OK & OK & OK \\
HKLNS shows a polyline from current location to the desired location & OK & OK & OK & OK & OK \\
Select "Click For Latest Info" & OK & OK & OK & OK & OK \\
Admin Update the information & & & & & \\
- Department information & OK & OK & OK & OK & OK \\
- Information for user & OK & OK & OK & OK & OK \\
\hline
\end{tabular}

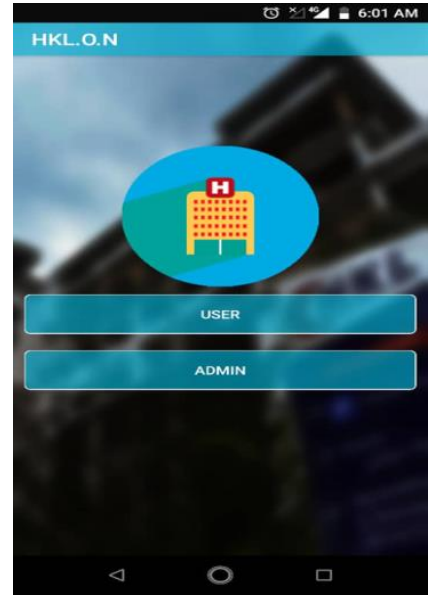

Figure 5. Main menu for HKLNS

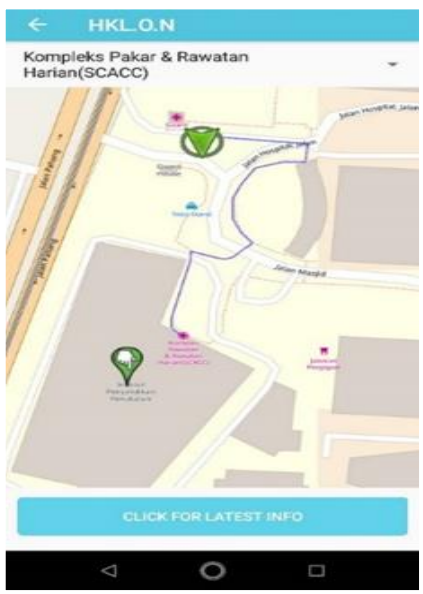

Figure 8. Selection to destination

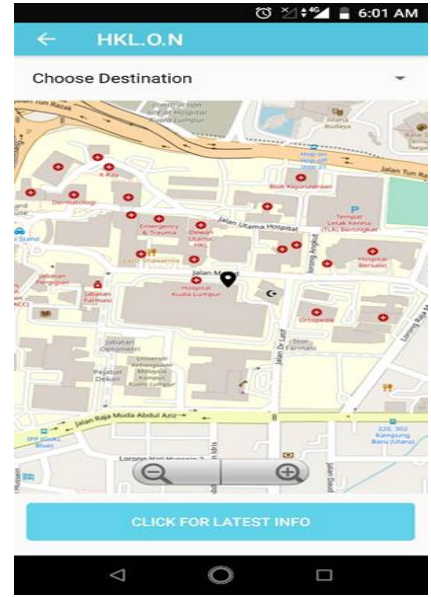

Figure 6. Interface detecting current location

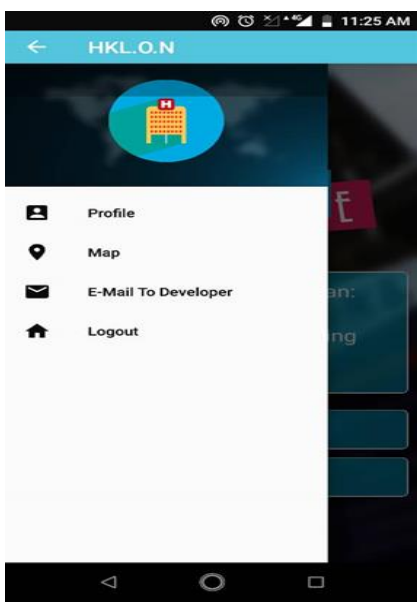

Figure 9. Admin menu

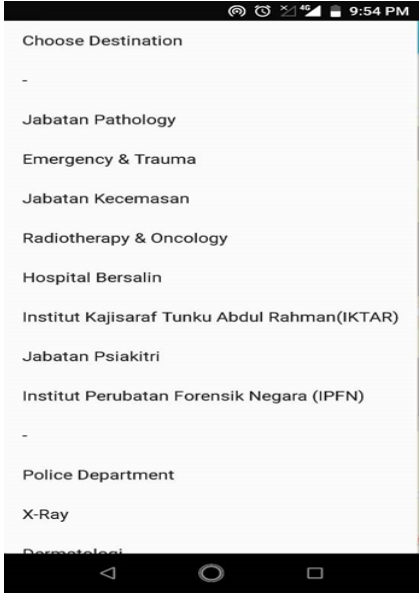

Figure 7. Choose desired department

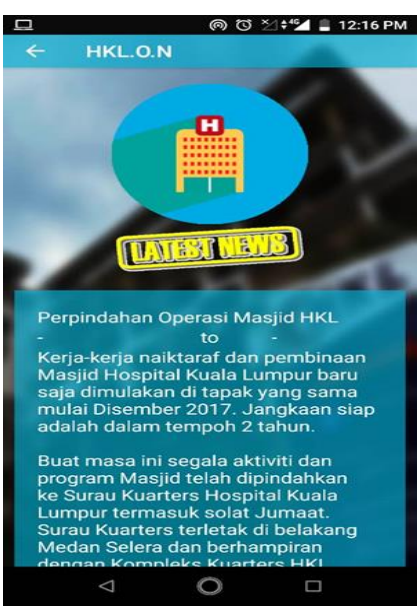

Figure 10. Latest news prompted

\subsection{Validation of system effectiveness testing}

Table 3 shows the result time taken before and after using HKLNS for 5 selected case studies. Every case study conducted shows more than $50 \%$ reduction of time taken from the current location to the desired 
location. As a result, it is shown that using mobile-based HKLNS enabled users to arrive at the desired departments in a shorter time.

In order to prove the hypothesis that the HKLNS was able to minimize the time taken to reach the desired department, t-test was measured with the $H_{0}$ : There is no significant difference in the experimental time taken using HKLNS and $H_{1}$ : There is a significant difference in the experimental time taken using HKLNS. The t-test result as in Table 4 indicates a two-tailed p-value of 0.00041 in which $p$-value $<0.05$. Therefore, $H_{0}$ is rejected with this sufficient evidence, significant (*p-value $\left.<0.05\right)$ and $H_{l}$ is supported as there is a significant difference in the reliability testing between with and without the HKLNS.

Table 3. Validation testing for HKL navigation system

\begin{tabular}{|c|c|c|c|c|c|c|}
\hline $\begin{array}{l}\text { Case Study } \\
\text { No. }\end{array}$ & Current Location & Desired Department & $\begin{array}{c}\text { Test } \\
\text { Method }\end{array}$ & $\begin{array}{l}\text { Time Taken } \\
(\min )\end{array}$ & $\begin{array}{l}\text { Difference } \\
\text { Time Taken }\end{array}$ & $\begin{array}{c}\text { Percentage Difference } \\
(\%)\end{array}$ \\
\hline 1. & $\begin{array}{c}\text { Kompleks Pakar } \\
\text { \& Rawatan Harian } \\
\text { (SCACC) }\end{array}$ & $\begin{array}{l}\text { Institut Urologi dan } \\
\text { Nefrologi (IUN) }\end{array}$ & $\begin{array}{l}\text { Before } \\
\text { After }\end{array}$ & $\begin{array}{l}5.03 \\
2.43\end{array}$ & 2.6 & 51.69 \\
\hline 2. & $\begin{array}{c}\text { Emergency \& } \\
\text { Trauma }\end{array}$ & $\begin{array}{l}\text { Radioterapi \& } \\
\text { Onkologi }\end{array}$ & $\begin{array}{l}\text { Before } \\
\text { After }\end{array}$ & $\begin{array}{l}5.37 \\
2.40\end{array}$ & 2.97 & 55.31 \\
\hline 3. & $\begin{array}{l}\text { Radioterapi \& } \\
\text { Onkologi }\end{array}$ & Hospital Bersalin & $\begin{array}{l}\text { Before } \\
\text { After }\end{array}$ & $\begin{array}{l}8.56 \\
4.21\end{array}$ & 4.35 & 50.82 \\
\hline 4. & Hospital Bersalin & $\begin{array}{l}\text { Insitut Perubatan } \\
\text { Forensik Negara } \\
\text { (IPFN) }\end{array}$ & $\begin{array}{l}\text { Before } \\
\text { After }\end{array}$ & $\begin{array}{l}9.02 \\
4.30\end{array}$ & 4.72 & 52.32 \\
\hline 5. & $\begin{array}{l}\text { Kompleks Pakar } \\
\& \text { Rawatan Harian } \\
\text { (SCACC) }\end{array}$ & $\begin{array}{l}\text { Jabatan Sajian } \\
\text { Diatetik }\end{array}$ & $\begin{array}{l}\text { Before } \\
\text { After }\end{array}$ & $\begin{array}{l}8.31 \\
4.04\end{array}$ & 4.27 & 51.45 \\
\hline
\end{tabular}

Table 4. Mann Whitney U test for without and with HKLNS

\begin{tabular}{ccc}
\hline \multicolumn{3}{c}{ Mann-Whitney U test of Two-Sample } \\
\hline \multirow{3}{*}{ Min } & Without HKLNS & With HKLNS \\
\cline { 2 - 3 } Max & 5.030 & 2.400 \\
Median & 6.020 & 4.300 \\
Mean & 7.840 & 3.235 \\
p-value & \multicolumn{3}{c}{$0.00041 *$} \\
\hline * $\boldsymbol{p}<\mathbf{0 5}$
\end{tabular}

\section{CONCLUSION}

This paper presents the outcome from outdoor navigation system that ease the visitors who went to Hospital Kuala Lumpur by mapping the hospital plan using OSM and GraphHopper API. The objective achieved and visitors were able to know their current location the location of the departments, navigate themselves to their desired department towards the accurate route in shorter time duration. In conclusion, Mobile-Based HKL Departments Navigation System (HKLNS) could give many advantages to the users. Furthermore, the research implemented GraphHopper API that support multiple routing algorithm such as the Dijkstra and $\mathrm{A}^{*}$ algorithm and the map use OSM API. The testing conducted included functionality testing and validation of system effectiveness testing. For future research, it is suggested for future researchers to work on the system's ability to give estimated time to arrive for user, to display direction guide for user to refer to and to provide a list of searches made.

\section{REFERENCES}

[1] M. W. Richey, J. L. Howard, E. W. Anderson, T. S. Logsdon, S. S. D. Jones, and W. E. May, "Navigation," Encyclopadia Britannica. Available: https://www.britannica.com/technology/navigation-technology. [Accessed Oct. 12, 2019].

[2] N. Hughes, J. Pinchin, M. Brown, and D. Shaw, "Navigating in large hospitals," in 6th International Conference On Indoor Positioning And Indoor Navigation, 2015, pp.13-16.

[3] Y. Li, M. Brown, J. Pinchin, and J. Blakey, "Comparison of methods to support in- hospital navigation a pilot study in healthy volunteers," in International Navigation Conference, 2015, pp. 1-7.

[4] N. Joibi, "Access to HKL Riddled With Challenges," The Star, October 7, 2013. [Online], Available: https://www.thestar.com.my/news/nation/2013/10/07/access-to-hkl-riddled-with-challenges-public-no-parkingspots-walkway-removed-and-bus-service-unre/ [Accessed June 25, 2018]. 
[5] P. L. J. Berry and S. Bell, S, "Pointing accuracy: does individual pointing accuracy differ for indoor vs. outdoor locations?," Journal of Environmental Psychology, vol. 38, pp. 175-185, 2014.

[6] R. Gallotti, M. A. Porter, and M. Barthelemy, "Lost in transportation: information measures and cognitive limits in multilayer navigation," Science Advances, vol. 2, no. 2, pp. 1-25, 2016.

[7] F. L. Greenroyd, R. Hayward, A. Price, P. Demian, and S. Sharma, "A tool for signage placement recommendation in hospitals based on way finding metrics," Indoor and Built Environment, vol. 27, no. 7, pp. 925-937, 2018.

[8] Khoo, V.-J. (2017), "Confusion arises as HKL outpatient services move to new facility," MIMS Today, April 6, 2017. [Online], Available: https://today.mims.com/confusion-arises-as-hkl-outpatient-services-move-to-newfacility. [Accessed July 5, 2018].

[9] E. Vilar, F. Rebelo, and P. Noriega, "Indoor human way finding performance using vertical and horizontal signage in virtual reality," Human Factors and Ergonomics in Manufacturing, vol. 16, no. 1, pp.61-81, 2014.

[10] S. Vasserman, M. Feldman, and A. Hassidim, "Implementing the wisdom of waze," in 24th International Joint Conference on Artificial Intelligence (IJCAI), 2015, pp.660-666.

[11] C. Vandeviver, "Applying google maps and google street view in criminological research," Crime Science, vol. 3, no. 1, pp. 13, 2014.

[12] K. A. F. A. Samah, N. Sabri, R. Hamzah, R. Roslan, N. A. Mangshor, and A. A. M. Asri, "Brute force algorithm implementation for traveljoy travelling recommendation system," Indonesian Journal of Electrical Engineering and Computer Science, vol. 16, no. 2, pp. 1042-1049, 2019.

[13] K. M. K. Weerakoon, K.S. Rupasinghe, T.P. Withanaarachchi, G.M.R.I. Godaliy adda, M.P.B. Ekanayake, "Outdoor human navigation with gps and sensor systems," in IEEE International Conference on Industrial and Information System (ICIIS), 2017, pp. 1-6.

[14] Y. Yun, J. Jin, N. Kim, J. Yoon, and C. Kim, "Outdoor localization with optical navigation sensor, imu and gp s," in IEEE International Conference on Multisensor Fusion and Integration for Intelligent Systems, 2012, pp. 377-382.

[15] I. Joo, J. Lee, C. Sin, S. Lee, and J. Kim, "Development and test of gps interference monitoring system," in International Conference on Control, Automation and Systems (ICCAS), 2014, pp. 1014-1017.

[16] G. M. Someswar, T. P. S. C. Rao, and D. R. Chiguruko, "Global navigation satellite systems and their applications," International Journal of Software and Web Sciences, vol. 3, no. 1, pp. 17-23, 2013.

[17] T. Khalifa, N. Sahar, N. Ramli, and M. Islam, "Circularly polarized microstrip patch antenna array for gps application,” Indonesian Journal of Electrical Engineering and Computer Science, vol. 15, no. 2, pp. 920-926, 2019.

[18] A. Badshah, N. Islam, D. Shahzad, B. Jan, H. Farman, M. Khan, and A. Ahmad, "Vehicle navigation in gps denied environment for smart cities using vision sensors," Computers, Environment and Urban Systems, vol. 77, pp. 1-11, 2018.

[19] P. K. Gaikwad and S. J. Pawar, "Implementation of real-time gps receiver system for providing navigation based services and sms tracking," in International Conference on Industrial Instrumentation and Control (ICIC), 2015, pp. 630-634.

[20] S. Yang, "Running head: google drive forensic analysis via api google drive forensic analysis via application programming interface," M.S. thesis, University of Central Oklahoma, 2015.

[21] X. Zhu, "Restful api for integrative literature information and knowledge service," M.S. thesis, University of Delaware, 2017.

[22] A. Sill, "When to use standards-based APIs (part 1)," IEEE Cloud Computing, vol. 2, no. 5, pp. 76-80, 2015.

[23] L. Palen, R. Soden, T. J. Anderson, and M. Barrenechea, "Success \& scale in a data-producing organization: the socio-technical evolution of openstreetmap in response to humanitarian events," in Proceedings of the 33rd Annual ACM Conference on Human Factors in Computing Systems, 2015, pp. 4113-4122.

[24] J. J. Arsanjani, P. Mooney, M. Helbich, and A. Zipf, "An exploration of future patterns of the contributions to openstreetmap and development of a contribution index," Transactions in GIS, vol. 19, no. 6, pp. 896-914, 2015.

[25] P. Neis and D. Zielstra, "Recent developments and future trends in volunteered geographic information research: the case of openstreetmap," Future Internet, vol. 6, no.1, pp.76-106, 2014.

[26] P. Welch, "Developing a commercial dynamic vehicle routing system-a case study," Open Door Logistics, July. 2017.

[27] V. Nallur, A. Elgammal, S. Clarke, "Smart route planning using open data and participatory sensing," in 11th International Conference on Open Source Systems (OSS), 2015, pp.91-100.

[28] N. Krismer, D. Silbernagl, M. Malfertheiner, and G. Specht, "Elevation enabled bicycle router supporting userprofiles," in 28th GI-Workshop on Foundations of Databases, 2016, pp. 74-79.

[29] K. A. F. A. Samah, B. Hussin, and A. S. H. Basari, "Modification of dijkstra's algorithm for safest and shortest path during emergency evacuation," Applied Mathematical Sciences, vol. 9, no. 31, pp. 1531-1541, 2015.

[30] S. K. Debnath, R. Omar, and N. Abdul Latip, "Comparison of different configuration space representations for path planning under combinatorial method," Indonesian Journal of Electrical Engineering and Computer Science, vol. 14, no. 1, pp. 1-8, 2019. 